\title{
Accessing causal relations in semantic memory
}

\author{
DANIELA B. FENKER \\ Otto von Guericke University, Magdeburg, Germany \\ and University of Göttingen, Göttingen, Germany \\ MICHAEL R. WALDMANN \\ University of Göttingen, Göttingen, Germany \\ and \\ KEITH J. HOLYOAK \\ University of California, Los Angeles, California
}

\begin{abstract}
Most studies investigating semantic memory have focused on taxonomic or associative relations. Little is known about how other relations, such as causal relations, are represented and accessed. In three experiments, we presented participants with pairs of words one after another, describing events that referred to either a cause (e.g., spark) or an effect (e.g., fire). We manipulated the temporal order of word presentation and the question participants had to respond to. The results revealed that questions referring to the existence of a causal relation are answered faster when the first word refers to a cause and the second word refers to its effect than vice versa. However, no such asymmetry was observed with questions referring to the associative relation. People appear to distinguish the roles of cause and effect when queried specifically about a causal relation, but not when the same information is evaluated for the presence of an associative relation.
\end{abstract}

Semantic memory is regarded as the long-term repertoire of our world knowledge (Tulving, 1972). Without world knowledge, we would be incapable of understanding the world around us and hence unable to communicate or to act in the service of goals (Hodges \& Patterson, 1997). Semantic memory contains knowledge about categories and features that we use to represent the world, as well as knowledge about relations between categories and features (see Murphy \& Medin, 1985). The great majority of this work has focused on either taxonomic relations (e.g., verifying category statements, such as "A robin is a bird") or general associative relations (e.g., priming a lexical decision about doctor by first presenting an associate such as hospital). Yet although taxonomic and associative relations are certainly important for understanding cognition, other types such as temporal, functional, and causal relations are also highly relevant for planning, predicting,

\footnotetext{
The experiments were conducted in partial fulfillment of the requirements for a PhD, which was obtained by the first author under the supervision of the second author at the University of Göttingen. The experiments were conducted during a visit of the first author at the Psychology Department of the University of California, Los Angeles, which was funded by the HSP III fellowship of the German Academic Exchange Service (DAAD). The study was also supported by BMBF Grant $01 \mathrm{GO} 0202$ (CAI). Portions of this research were presented at the 2001 annual meeting of the Psychonomic Society, Orlando, FL; the 2002 conference of the Deutsche Gesellschaft für Psychologie, Berlin; and the 2004 International Psychology Congress, Beijing. Correspondence concerning this article should be addressed to D. B. Fenker, Otto von Guericke University, Department of Neurology II, Leipziger Str. 44, 39120 Magdeburg, Germany (e-mail: sellner@neuro2.med.uni-magdeburg.de).
}

acting, and reasoning. Research on categorization has focused increased attention on causal relations (e.g., Lien \& Cheng, 2000; Murphy, 2002; Murphy \& Medin, 1985; Rehder, 2003; Waldmann, 1996; Waldmann, Holyoak, \& Fratianne, 1995); nonetheless, few studies have addressed the question of how causal and similar functional relations are stored and accessed in semantic memory (but see Krüger, Nuthmann, \& van der Meer, 2001; Moss, Ostrin, Tyler, \& Marslen-Wilson, 1995; Tyler \& Moss, 1997; van der Meer, Beyer, Heinze, \& Badel, 2002).

The existence of multiple relations within semantic memory raises a particularly interesting question that has been neglected by theories of semantic knowledge: Assuming that different types of relational knowledge are relevant in different contexts, how are specific relations accessed within a network that contains many different kind of relations? The main goal of our study was to address this question by focusing on a particularly important class of relations, those that are causal in nature.

\section{Causal Relations}

The nature of causality has long been a hotly debated topic in philosophy. However, one aspect of causal relations seems undisputed: They are asymmetric (Hausman, 1998; Pearl, 2000; Waldmann, 1996). Causes temporally precede and generate effects in the world. For example, smoking causes lung cancer but lung cancer does not cause smoking.

There has been sharp disagreement among psychologists as to whether this asymmetry is mirrored in human cognitive representations. Some researchers in the area of 
learning have claimed that causal asymmetry is not a feature that is represented when people learn about causal relations (Cobos, López, Cano, Almaraz, \& Shanks, 2002; Shanks \& López, 1996). According to this associative view, learning leads to knowledge about the associative strength between cues and outcomes. Although associative relations are also asymmetric, being directed from cues to outcomes, this asymmetry is dependent on the temporal assignment of learning events to the roles of cues and outcomes, and not on causal asymmetry. Causes and effects can arbitrarily function as cues or outcomes, depending on the temporal order of events in a learning task. By contrast, an alternative account, the causal-model theory, has postulated that learners explicitly represent asymmetric causal relations and use this knowledge in learning (Waldmann, 1996, 2000, 2001; Waldmann \& Holyoak, 1992; Waldmann et al., 1995).

The debate between the associative view and the causalmodel theory has focused on learning. However, similar questions can be asked with respect to the results of learning about knowledge stored in semantic memory. According to the associative view, asymmetries in retrieval time of semantic causal knowledge should be reducible to asymmetries of the strength of associative links, whereas a causalmodel view would posit that retrieval is in part determined by access to specifically causal relational knowledge. The main goal of the present research is to derive discriminating predictions from these two competing paradigms with respect to how causal relations are accessed in semantic memory, and to test these predictions.

\section{Asymmetry in Recognition of Causal Relations}

The present research uses a paradigm that focuses on the retrieval of existing causal knowledge, rather than learning new causal relations. We used a relation recognition paradigm in which we presented participants with pairs of words one after another. On critical trials, each word referred to an event that acted as a cause (e.g., spark) or to an effect (e.g., fire). We manipulated the temporal order in which the words were presented and the question to which participants had to respond. In the causal conditions, we asked participants to judge as quickly as possible whether the two mentioned events were causally related. We compared two types of causal conditions. In the predictive condition, the cause (e.g., spark) was presented temporally prior to the effect (e.g., fire), whereas in the diagnostic condition the order of presentation was reversed. Regardless of the ordering, the correct answer would be "yes" in both conditions.

Events such as spark and fire can be connected by a number of different relations (e.g., spatial, temporal, associative); accordingly, assessing whether a causal relation exists between them requires access to the specific set of causal relations stored in semantic memory. Since causal relations are asymmetric, it does not suffice to check whether the two events are merely linked; rather, it is necessary to map them to the roles of cause and effect and check whether they are connected by a causal relation. Causal model theory predicts that this mapping process will lead to longer recognition latencies for diagnostic versus predictive causal relations. A key assumption driving this prediction is that people (and other animals) typically experience causes temporally prior to their effects. Because we normally experience causes prior to their effects, we assume that we have an automatic tendency to infer that when one causal event is mentioned temporally prior to another, the former event is the cause and the latter is its effect (a kind of "congruity" effect). For diagnostic relations, this leads to a mismatch between the temporal order of experienced events and causal order stored in semantic memory, which according to our analogical mapping account leads to longer recognition times (see below). Evidence consistent with this prediction of a congruity effect for temporal relations was reported in a recent study by van der Meer et al. (2002), which showed that a text is easier to understand when the temporal order in which events are mentioned in the text corresponds to that in which they occur in the real world. In a related study, participants read a series of word-pairs describing sequenced events (Krüger et al., 2001). The task was to judge whether the two words within an item were semantically related (i.e., part of a typical sequence). Similar to the van der Meer et al. study, this experiment also showed that judgments were made more quickly when information followed the temporal arrow rather than being ordered in the reverse direction.

A variety of specific mechanisms, such as the iconicity account (Zwaan \& Yaxley, 2003), explains such a congruity effect. According to this account, internal representations mirror spatial and temporal properties of domains. However, it is questionable whether this approach could explain the flexible access of associative versus causal relations with identical items (see Experiment 3). Another possible mechanism involves analogical mapping. In order to assign events to roles in a causal relation, each item in a pair must be mapped to a causal role. According to at least one major model of analogical mapping, Learning and Inference with Schemas and Analogies (LISA; see Hummel \& Holyoak, 1997, 2003), mapping is based on distributed semantic representations. It will be facilitated to the degree that the semantic features of a presented item overlap with the features of the role to which the item maps. Given the assumption that the semantic code for temporally prior overlaps with the semantic code for cause, whereas the code for temporally after overlaps with that for effect, recognition of a causal relation will be facilitated when the cause is introduced temporally prior to the effect (rather than vice versa), yielding a congruity effect of the form predicted by causal-model theory.

\section{Dissociating the Causal Model From the Associative View}

Causal model theory predicts faster access for predictive than for diagnostic relations because of the underlying asymmetry of causal directionality. Nonetheless, associative theories could potentially predict faster ac- 
cess to predictive than to diagnostic items in a relation detection task by assuming that associations in the predictive direction may tend to be stronger than associations in the reverse direction. If one assumes that associative relations are to a large extent based on the experience of spatiotemporal contiguity of event pairs in the real world or in texts, it is plausible to assume that people encounter event pairs more often in the predictive than in the diagnostic direction. Friedman (1990) reported studies showing that autobiographical memories are typically reported in chronological order. Moreover, in a developmental study, Friedman (2002) found that 4- and 8-month-old children showed a significant preference for the forward as opposed to the reverse presentation of a video in which water was poured into a glass.

However, experienced temporal order is not the only factor that influences the strength of association. For example, although sex temporally precedes pregnancy, the statistical contingency between pregnancy and sex is certainly stronger than vice versa. Thus, association strength is also affected by the number and frequency of alternatives in each direction (i.e., statistical strength).

In order to dissociate the predictions of the causal-model versus associative views, in the present study we used materials for which the association strength between cause and effect was equated for the predictive and the diagnostic directions. Although it is likely that predictive relations in general tend to be associated more strongly than diagnostic relations, it is possible to find items in which both associations are roughly equal. According to the associative view, there should be no difference in access time for these items in the two directions when associative strength is controlled. In contrast, the causal-model view predicts that the mismatch between order of presentation and causal directionality should lead to faster access for predictive relations than for equally associated diagnostic relations.

\section{EXPERIMENT 1}

The main goal of Experiment 1 was to test our prediction that predictive relations would be accessed more quickly than diagnostic relations even for items that were equally associated in both directions. The stimulus pairs were selected on the basis of the USF Word Association Norm list (Nelson, McEvoy, \& Schreiber, 1998) first and then underwent an additional norming study (see Method). Since statistical strength is an important factor affecting associative strength for causal items, we used the norming study to derive causally related item pairs that were equated in both directions in terms of the strength of statistical relations.

\section{Method}

Participants and Design. Twenty-six undergraduate students from the University of California, Los Angeles, participated for course credit. Their vision was normal or corrected to normal. Three participants had to be replaced ( 1 due to computer failure, 2 with an overall error rate above $20 \%$ ). The stimuli were presented in a within-subjects design. Every participant saw every word pair once; the order of the trials and the type of stimuli within a trial (predic- tive vs. diagnostic order) were randomized and counterbalanced, respectively.

Stimuli. The stimulus materials consisted of 68 causally related word pairs (e.g., moon-tide) and 68 weakly associated filler word pairs (e.g., ring-emerald; see the Appendix for the item list). The causal and the associated filler word pairs shared the same low strength of association in each direction (Table A1). The word pairs were selected from the USF Word Association Norms (Nelson et al., 1998). In that study, participants were asked to write down the first word that came to mind that was meaningfully related to or strongly associated with a presented word. Each participant produced only a single word. Nelson et al. calculated the forward and backward strength between the cue and the target word for each of 5,019 words. The forward strength (FSA in Table A1) was calculated as the number of participants who produced a particular target word (for a given cue) divided by the number of participants in that group. The backward strength was calculated the same way, except that now the target word served as the cue word (BSA in Table A1). The strength of association between the two words is represented by a number from 1.0 to 0 for each direction. We chose word pairs from this database that were connected by a causal relation. These word pairs had a low strength of association (.00-.2) in both directions (predictive/forward and diagnostic/backward). Only weakly associated words were chosen, to reduce the possible influence of strength of association as much as possible. A total of 250 word pairs were selected.

As a next step, we conducted an additional norming study to account for the strength of statistical contingency, an important factor affecting associative strength between causal items. We presented these 250 word pairs to 80 participants (UCLA undergraduates) in a questionnaire task. Both causal and filler word pairs were presented, and for each pair the students were asked to judge whether there was a causal relation between the presented words. If the students detected a causal relation, the next step for them was to imagine that the event described by the first of the two words occurred 100 times. Their task was to estimate the conditional frequency for the event described by the second word (e.g., "fire occurs 100 times; how often does heat occur?"). They indicated their rating on a scale from 0 to 100 , with increments of 10 . The order of items was randomized within the questionnaire and the version (diagnostic or predictive) of each item was randomly chosen and counterbalanced over two versions of the questionnaire.

On the basis of the results of the norming study, we selected 68 pairs for our experiments that did not differ by more than 30 mean rating points between the two directions (predictive and diagnostic). Table A1 shows the difference of the ratings for the individual items (FD). The rating difference between the two directions summed up to 0 over all selected word pairs, which means that the overall conditional frequency rating for predictive causal relations was equated with the overall frequency rating for the diagnostic causal relations. The associated filler word pairs did not share a causal relation but were roughly equal to the causal items in terms of bidirectional strength of association (see Table A1).

In the reaction time (RT) experiment, the test pairs were presented in the font Arial Black, point size 24, on a white background. All experiments were programmed in Superlab and implemented on Macintosh iMac computers connected to a 15 -in. screen with $1,026 \times$ 768 pixel resolution and 256 colors. The words were created as pct files in Canvas 6.0 graphics software.

Procedure. The participants were shown written instructions. They were asked to decide whether there was a causal relation between the events described by the two words presented on the computer screen. To make it clear that we wanted the participants to assess the existence of a causal relation independently of the sequence of the item pairs, we additionally specified that the task was to assess "whether the event described by the first word causes or is caused by the event described by the other word." After reading the instructions, the participants in this and the other experiments were 
asked to repeat them in their own words to avoid any misunderstanding about their task.

At the beginning of each trial, the participants saw a fixation cross in the center of the screen. After $1,000 \mathrm{msec}$, the cross disappeared and a blank screen was presented for $500 \mathrm{msec}$. The first word of the item pair was then presented for $1,000 \mathrm{msec}$, followed by the second word (which replaced the first word). Thus, the interstimulus interval (ISI) was 0 and the stimulus onset asynchrony (SOA) was $1,000 \mathrm{msec}$. The choice of SOA was motivated by van der Meer's (1999) finding that a 1,000-msec SOA yielded a stronger effect than a shorter SOA did. The second word remained on the screen until the participant pressed one of the two response keys. If participants viewed the item as causal, they were requested to press the letter "C" on the keyboard; otherwise they were to press the letter "N." Each participant completed 136 trials. Of the 68 causal trials, half were presented in the predictive direction (cause-effect) and half were presented in the diagnostic direction (effect-cause); the remaining 68 trials were noncausal filler word pairs. The order of the filler word pairs was also counterbalanced over participants. The RT for each trial was measured and recorded by the Superlab software. The program also recorded errors.

The participants were required to respond to 20 practice trials (10 causal, 10 filler) at the beginning of the session. They received feedback in the practice trials; during the experimental trials, no feedback was given.

\section{Results and Discussion}

The most important findings concern RTs for the predictive and diagnostic causal items. In all experiments, the analysis of RTs included only trials that were answered correctly, excluding outliers that were more than two standard deviations above or below the participant's individual mean within each condition. On average, this criterion led to the exclusion of two to three trials per participant.

Overall, RTs were $68 \mathrm{msec}$ faster for predictive trials (mean RT of $1,016 \mathrm{msec}$ ) than for diagnostic trials (mean RT of 1,084 msec). The mean RT of the fillers was $1,083 \mathrm{msec}$. A paired sample $t$ test revealed that the advantage for the predictive trials was significant $[t(25)=2.64$, $p=.01$, with a small to medium effect size of $d=.34$. Error rates for the predictive and diagnostic pairs were $15 \%$ and $18 \%$, respectively, and $11 \%$ for fillers, and did not differ significantly $[t(25)=1.74, p=.09]$. An additional regression analysis was performed using causal direction, statistical frequency from our norming study, and strength of association (USF) as predictor variables and RTs as a dependent variable. This analysis revealed causal direction as the sole significant predictor for RT $(p<.01)$. Statistical frequency and strength of association did not predict RTs significantly ( $p=.42$ and $p=$ .82 , respectively).

The results confirm our hypothesis that causal relations are accessed faster when the order of presented events follows the predictive rather than the diagnostic order. These results support the hypothesis that causal direction influences the retrieval of causal relations. The analysis of errors revealed no speed-accuracy trade-off between the different conditions. The advantage of predictive over diagnostic orderings favors the causal-model view over the associative account because the effect was found with items that had symmetric association strengths in both presentation orders. Thus, the difference in RTs cannot be explained by asymmetries of associative strength.

\section{EXPERIMENT 2}

In Experiment 1 the predictive and diagnostic items were presented within a single block in a random order. Although we explicitly instructed participants that both predictive and diagnostic relations are part of the general class of causal relations, there may be a tendency to interpret a request to check for causal relations as primarily directed toward the more natural predictive direction. Participants may therefore first check for the predictive direction before considering the possibility of a diagnostic relation, a strategy that would yield longer RTs for diagnostic items. To rule out the possibility that participants primarily interpret causal questions as requests to assess predictive relations, we presented predictive and diagnostic items in separate blocks and explicitly specified the access direction in the task instructions. In two different blocks, participants had to judge either whether the first word described the cause of the second event (predictive block) or whether the first word described an effect of the second event (diagnostic block). As in Experiment 1, the filler items were weakly associated words. The item pairs in the predictive block were either causally related (predictive order) or associated, whereas the item pairs in the diagnostic block were either causally related (diagnostic order) or associated.

\section{Method}

Participants and Design. Twenty-eight UCLA undergraduates with normal or corrected-to-normal vision received course credit for their participation. We replaced 4 participants because their overall error rate exceeded $20 \%$. For the 4 replaced participants, the false alarm rate for the items that were associated but not causally related was particularly high, indicating that these participants were biased toward a causal response and therefore did not clearly fulfill the task requirement to differentiate between word pairs that were causally related rather than only associated.

The stimuli were presented in a within-subjects design; every participant saw every word pair once. The order of the two blocks and the version of the stimuli assigned to either block (predictive vs. diagnostic) were counterbalanced, creating four different counterbalanced versions.

Stimuli. The stimulus material consisted of 64 causal and 64 associated filler word pairs that were used in Experiment 1. We excluded four causal word pairs that were consistently answered incorrectly in Experiment 1, in order to reduce the error rate and decrease noise in the data. Their removal did not alter the overall balanced strength of association ratings. The overall statistical frequency was virtually symmetric, with a minuscule bias toward the diagnostic direction $(\mathrm{FD}=-1)$. For each block, a list of stimuli was created that contained 32 causal word pairs in one of the two directions and 32 associated filler word pairs.

Procedure. Half of the participants received the predictive block first and the diagnostic block second; the other half were given the blocks in the opposite order. In the predictive block, participants were asked to judge whether or not the first word described the cause of the event represented by the second word, whereas in the diagnostic block, they had to decide whether the first word described the effect of the event represented by the second word. Prior to each block, the 
participants were given 10 practice trials ( 5 predictive and 5 filler trials before the predictive block, and 5 diagnostic and 5 filler trials before the diagnostic block), for which feedback was given.

\section{Results and Discussion}

The results replicated the pattern observed in Experiment 1. Mean RT was significantly shorter (by $69 \mathrm{msec}$ ) in the predictive condition $(817 \mathrm{msec})$ than in the diagnostic condition $(886 \mathrm{msec})$. The overall analysis showed a significant effect of the factor causal direction $[F(1,26)=$ $\left.5.39, M S_{\mathrm{e}}=12,542.17, p=.02\right]$, with an effect size of $\eta^{2}=.18$. The order of the blocks exhibited neither a significant main effect $\left[F(1,26)=0.26, M S_{\mathrm{e}}=39,206.34\right.$, $p=.61]$, nor a significant interaction with causal direction $\left[F(1,26)=0.28, M S_{\mathrm{e}}=12,542.17, p=.60\right]$. The mean RT for the filler trials was $848 \mathrm{msec}$. The error rate was $13 \%$ for the fillers, $13 \%$ in the predictive block, and $12 \%$ in the diagnostic block; the difference between the predictive and the diagnostic blocks did not approach significance $[t(27)=.80, p=.43]$. As in Experiment 1 , a regression analysis revealed causal direction as the only significant predictor for RTs $(p<.01)$. The other factors did not significantly contribute to the RT differences ( $p=.09$ for statistical frequency, and $p=.19$ for strength of association).

Experiment 2 thus replicated the results of Experiment 1 using a design in which predictive and diagnostic items were blocked with specific instructions to check for either a predictive or a diagnostic relation. Moreover, each block was preceded by practice trials. Accordingly, participants were clearly aware of the fact that in the diagnostic block they would be confronted only with diagnostic items and that their task was to say "yes" when they saw a diagnostic relation. Nonetheless, RTs were slower for the diagnostic than for the predictive block. Thus the detrimental effects of a mismatch between the order of presentation and causal order is not a simple bias that can be remedied by instructions and blocking of trials; rather, it seems to be an automatic consequence of the mechanism by which the cognitive system accesses causal knowledge stored in semantic memory.

\section{EXPERIMENT 3}

Experiments 1 and 2 tested the causal-model view against the associative view by asking participants to access causal relations using pairs of items that had symmetric association strengths in both causal directions. Experiment 3 extended the relation verification paradigm by asking half of the participants to check whether the events described in an item pair were causally related and the other half whether they were associated. In terms of causal-model theory, representing and evaluating a causal relation requires a representation in which each event is mapped to a specific role, the cause or the effect. This mapping will be made more quickly if the temporally prior item is the cause, yielding shorter RTs in the predictive than in the diagnostic presentation order. In contrast, the general rela- tion associated does not differentiate the roles of the two items, so no such mapping process is required. Accordingly, causal-model theory predicts an interaction, such that the advantage of the predictive over the diagnostic ordering should be observed only for queries about causality, and not for queries about association. In contrast, the associative view does not predict an order effect for either query (given that pairs are selected to have symmetric association strengths).

\section{Method}

Participants and Design. Forty-four UCLA undergraduate students participated in this experiment and received course credit. Their vision was normal or corrected to normal. Half of this group was assigned to the causal and half to the associative condition. Two participants in the associative and 3 in the causal condition had to be replaced because of high overall error rates $(>20 \%)$.

Stimuli. All 44 participants saw causal word pairs and unrelated filler word pairs. In both the causal and the associative conditions, the participants were presented with 64 causal and 64 unrelated filler word pairs. The causal items were the stimuli used in Experiment 2. The unrelated filler word pairs were made up of single words from causal relations that we did not select for the previous studies due to excessively divergent ratings in the norming study. The words were combined randomly to avoid any semantic or associative relationships. As in the earlier experiments, the causal items were presented in either the predictive or the diagnostic direction, with the versions of the items being counterbalanced across participants.

The critical causal and associated word pairs were the stimuli used in the previous experiments. In the associative condition, we also included some associated but noncausal items (e.g., emerald-ring, vehicle-bicycle). Additional unrelated items were also included in this condition to equate the proportion of correct "yes" and "no" responses. The associative condition included 64 causal items, 32 associated but noncausal items, and 96 unrelated filler word pairs (see Table A2 in the Appendix for the list of unrelated words). We added noncausal, weakly associated item pairs to the associative condition to ensure that participants in this condition did not become aware of the fact that all items were causally related. Our goal was to ensure that participants focused on associative relations rather than causal relations. To avoid a "yes" bias, we added unrelated items in the associative condition. This design led to a greater number of items in the associative condition, but allowed us to compare the judgments to an identical set of causal pairs across the two conditions, which was the principal goal of our experiment.

Procedure. In the causal condition, the participants were requested to judge whether there was a causal relation (as in Experiment 1). In the associative condition, the participants were instructed that their task was to judge whether "there is an association between the two words." They were told to press the "A" key if the words were "related in some meaningful way," and to press the "N" key if there was no relation. In other respects, the procedure was the same as in the previous experiments.

\section{Results}

Figure 1 displays the mean RTs for the causal items for both the causal and the associative instructions. As in the previous experiments, with causal instructions diagnostic items led to slower RTs than did predictive items. In contrast, with associative instructions the very same items were responded to equally quickly in both orders. The analysis of the design for the within-subjects factor of causal direction (predictive vs. diagnostic) and the between-subjects factor 


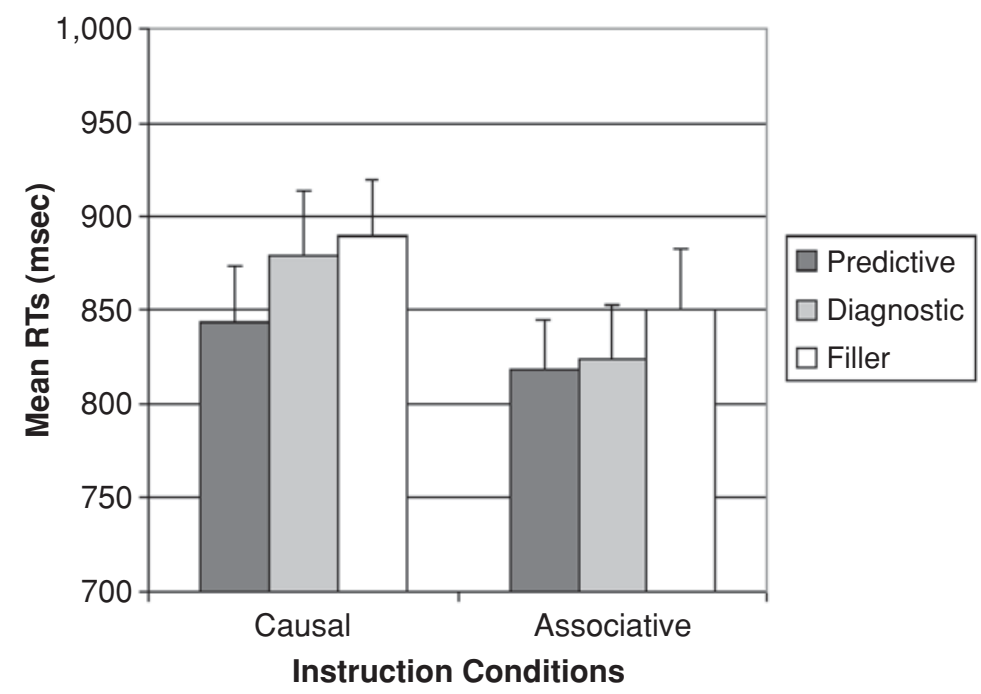

Figure 1: Mean reaction times (RTs) and standard errors (error bars) for causal stimuli and filler items in the causal and associative conditions for pairs presented in the predictive and diagnostic orders (Experiment 3 ).

type of instruction (causal vs. associative) yielded a significant effect for causal direction $\left[F(1,42)=7.81, M S_{\mathrm{e}}=\right.$ $1,231.4, p<.01]$, with an effect size of $\eta^{2}=.16$. There was no significant main effect of instruction $[F(1,42)=$ $\left..94, M S_{\mathrm{e}}=39,283.3, p=.34, \eta^{2}=.02\right]$. The interaction for the two factors was marginally significant $[F(1,42)=$ $\left.4.03, M S_{\mathrm{e}}=1,231.4, p=.05\right]$, with a small effect size of $\eta^{2}=.09$.

A post hoc analysis using the Scheffé test showed a significant difference between the predictive and diagnostic causal word pairs for the causal instruction $(p<.05)$. The difference for the causal word pairs for the associative instruction was not significant $(p=.96)$. In order to check that this null effect of the causal items in the associative task was not due to lack of power, we calculated the power employing a one-sided assumption using the standard error of the mean (11.74) for the associative condition and the difference of the means for the causal condition $(36 \mathrm{msec})$. A power of .92 was obtained, which was above the minimum acceptable power of .80 (Cohen, 1988). The diagnostic items in the causal condition led to longer RTs than both the diagnostic items $(p<.01)$ and the predictive items $(p<.01)$ in the associative condition. In contrast, RTs for the predictive items in the causal condition were not significantly different from those produced by either type of item in the associative conditions.

We also conducted an analysis of the errors. The error rates for the predictive and diagnostic items were $11 \%$ and $15 \%$, respectively, in the causal condition, and $13 \%$ and $14 \%$, respectively, in the associative condition. The fillers yielded an error rate of $3 \%$ in both conditions. An analysis of variance revealed an effect of causal direction $\left[F(1,42)=4.57, M S_{\mathrm{e}}=3.78, p=.04\right]$, with an effect size of $\eta^{2}=.09$. No other factor was significant. Finally, the regression analysis showed that for the causal condition, causal direction was a reliable predictor of RT differences $(p<.01)$-in contrast to statistical frequency $(p=.65)$ and strength of association $(p=.92)$. Moreover, the regression analysis for the associative condition confirmed that causal direction ( $p=.94)$, statistical frequency ( $p=$ $.74)$, and strength of association ( $p=.46)$ were not significant predictors of the RTs.

\section{Discussion}

The main finding in Experiment 3 was that participants proved capable of task-specific access to different types of relations when queried in different ways about an identical set of causally related items. When participants were asked to judge whether a causal relation was present, the usual RT advantage of predictive over diagnostic relations was found. However, for the identical items, access was equally fast regardless of causal direction when the instructions asked participants to judge whether the words within the item pairs were associated. This interaction supports the assumption of causal-model theory that mapping into causal roles is required for assessing a causal relation, but not for assessing an associative relation. In addition, the equal RTs obtained for associative judgments in the two directions confirm that our norming procedure succeeded in equating associative strength across directions for causally related word pairs.

RTs for diagnostic items in the causal condition were longer than all the other RTs. In contrast, the predictive items in the causal condition yielded RTs comparable to those for both the diagnostic and the predictive items in the associative condition. These results indicate that access to predictive causal relations is accomplished about as quickly as the determination of a general associative relationship between concepts.

Experiment 3 provides the most convincing evidence against the view that recognition latencies are generally driven by strength of association. Some might argue that free association norms or assessments of statistical strength might not be sensitive enough to asymmetries 
of associative relations. However, this hypothesis would predict equal effects for associative and causal queries, which is inconsistent with the obtained interaction. An alternative view might be that the asymmetry between predictive and diagnostic item pairs in Experiments 1 and 2 is driven by temporal order of events in the real world or in natural language. However, this hypothesis also fails because it would predict identical effects for associative and causal queries. Our findings uniquely support the view that people are capable of selectively accessing causal and associative relations, two types of relations that cannot be reduced to each other. ${ }^{1}$

\section{GENERAL DISCUSSION}

\section{Summary}

The three experiments reported here reveal that causal relations are retrieved from semantic memory and evaluated more easily in the predictive cause-effect order than in the reverse diagnostic effect-cause order. We have demonstrated this effect with materials that had equal association strength in both directions, assuring that the effect is not driven by asymmetries of association strength. In addition, we were able to show that the RT advantage for the predictive order persisted even when participants knew in advance what type of causal relation (predictive or diagnostic) they were going to see. The latter result indicates that this type of congruity effect is not due to the interpretation of causal relations as referring primarily to predictive relations, but is, rather, grounded in a deeper characteristic of causal semantic memory.

The overall RTs obtained in Experiment 1 were higher than those in Experiment 2. This difference may reflect the fact that in Experiment 2 participants knew in advance whether they were going to see a predictive or a diagnostic relation, reducing the amount of processing required on each individual trial.

In Experiment 3, we were able to demonstrate a dissociation between retrieval and evaluation of causal relations versus general associative relations. In particular, the RT advantage of pairs in the predictive as opposed to the diagnostic order disappeared when participants were asked to assess whether the items were associated, rather than causally related. This dissociation is consistent with the hypothesis that assessing causal relations requires mapping of the two events into specific causal roles ("cause" and "effect"), a process that is facilitated in the predictive order because the semantic features of the "temporally prior" event overlap with those of the "cause" role, whereas the features of the "temporally after" event overlap with those of the "effect" role (Hummel \& Holyoak, 1997, 2003). In contrast, the general "association" relation does not distinguish the roles of the two items in a pair; hence temporal order of presentation no longer has an effect on decision time. The present findings raise problems for theories that treat causal relations as simply instances of general associative relations (Shanks \& Dickinson, 1987), and they demonstrate our capacity to selectively access causal versus associative relations.

\section{Further Implications}

Our results are compatible with the assumptions of relational element theory (Chaffin, 1992; Chaffin \& Herrmann, 1987; Herrmann \& Chaffin, 1986). For example, a causal relation would be composed of temporal and spatial contiguity, as well as the element of causal directedness. Hence, in order to determine a causal relationship between two concepts, the elements of the connection between these concepts need to be evaluated, with a primary emphasis on causal direction that is reflected in asymmetric response times between the two directions. In contrast, the determination of an associative relation puts fewer constraints on the evaluation process, yielding equal retrieval times for both causal directions.

In the present study, we found that predictive causal relations can be accessed faster than diagnostic ones. One interesting research question is whether this effect can be reduced, or perhaps even reversed, with expertise. Physicians, for example, are confronted with diagnostic questions all day long, so it seems likely that they will learn to access knowledge in the diagnostic direction very efficiently. Future research should address the question of whether the process of accessing and evaluating causal relations changes with diagnostic expertise.

Experiment 3 demonstrated a dissociation between causal and associative relations. Causal relations are a type of basic semantic relation in which the entities being related play specific roles (in contrast to associative relations, which do not distinguish roles). Other semantic relations may show similar dissociations. For example, Spellman, Holyoak, and Morrison (2001) have shown that several basic semantic relations (e.g., category membership, antonymy) play a role in priming lexical access. Some of these, such as category membership, clearly distinguish specific roles (e.g., all canaries are birds is true, but all birds are canaries is false, reflecting the asymmetry of the roles of "instance" and "category"). The paradigm used in the present study could be readily adapted to investigate the cognitive representations of other relations in semantic memory. It is important to identify both the commonalities and differences among the varied semantic relations that constitute our knowledge of the world.

Our main goal was to measure how causal and associative relations are accessed in semantic memory. A related line of research has focused on the question of how causal relations are accessed in mental models that are formed as a consequence of text comprehension (see Bloom, Fletcher, van den Broek, Reitz, \& Shapiro, 1990; Graesser, Singer, \& Trabasso, 1994). Graesser et al. (1994) showed that causal antecedents are activated more readily than causal consequences, a result that might seem to contradict our finding that access to diagnostic relations is slower relative to predictive relations. However, there are important differences between the two experimental paradigms. In particular, inferring missing cause information is a prerequisite to understanding texts, whereas predictions of future events in the story are typically optional.

Moreover, diagnostic inferences need to be distinguished from diagnostic order. It is possible to learn about 
events in the predictive order and later reason backward in time on the basis of effect information (i.e., to make diagnostic inferences). In contrast, in diagnostic learning, or diagnostic semantic memory tasks such as that used in the present study, the order of presentation of events contradicts the natural order. It has been shown that text understanding is also slowed when the order of mentioned events contradicts the underlying temporal order that is being described (Rinck, Hahnel, \& Becker, 2001), a finding that is consistent with our findings.

A related important research question concerns the differentiation between temporal and causal relations (see Krüger et al., 2001; van der Meer et al., 2002). Temporal knowledge in semantic memory typically concerns events that regularly precede each other. Since Hume's $(1739 / 1978)$ classic analysis of causality, temporal precedence paired with regularity of succession has been viewed as one of the main characteristics of causality. For example, fire does not only precede smoke, it regularly precedes smoke, which is an empirical indicator of an underlying causal relation. It may be possible to distinguish temporal from causal relations by using episodic tasks (e.g., text comprehension), which make it possible to present singular temporal successions. It would also be interesting to investigate different types of causal relations, such as those based on social conventions.

\section{REFERENCES}

Bloom, C. P., Fletcher, C. R., van den Broek, P., Reitz, L., \& ShaPIRO, B. P. (1990). An on-line assessment of causal reasoning during comprehension. Memory \& Cognition, 18, 65-71.

Chaffin, R. (1992). The concept of semantic relation. In A. Lehrer \& E. Kittay (Eds.), Frames, fields, and contrasts: New essays in semantic and lexical organization (pp. 253-288). Hillsdale, NJ: Erlbaum.

Chaffin, R., \& Herrmann, D. J. (1987). Relation element theory: A new account of the representation and processing of semantic relations. In D. Gorfein \& R. Hoffmann (Eds.), Memory and learning: The Ebbinghaus Centennial Conference (pp. 221-245). Hillsdale, NJ: Erlbaum.

Cobos, P. L., López, F. J., Cano, A., Almaraz, J., \& Shanks, D. R (2002). Mechanisms of predictive and diagnostic causal induction. Journal of Experimental Psychology: Animal Behavior Processes, 28, 331-346.

COHEN, J. (1988). Statistical power analysis for the behavioral sciences (2nd ed.). Hillsdale, NJ: Erlbaum.

Friedman, W. J. (1990). About time. Cambridge, MA: MIT Press.

Friedman, W. J. (2002). Arrows of time in infancy: The representation of temporal-causal invariances. Cognitive Psychology, 44, 252-296.

Graesser, A. C., Singer, M., \& Trabasso, T. (1994). Constructing inferences during narrative text comprehension. Psychological Review, 101, 371-395.

Hausman, D. (1998). Causal asymmetries. Cambridge: Cambridge University Press.

Herrmann, D. J., \& Chaffin, R. (1986). Comprehension of semantic relations as a function of the definition of relations. In F. Klix \& H. Hagendorf (Eds.), Human memory and cognitive capabilities: Mechanisms and performance (pp. 311-319). Amsterdam: Elsevier, North-Holland.

Hodges, J. R., \& Patterson, K. E. (1997). Semantic memory disorders. Trends in Cognitive Sciences, 1, 67-72.

Hume, D. (1978). A treatise of human nature. Oxford: Oxford University Press, Clarendon Press. (Original work published 1739)
Hummel, J. E., \& HolyoAK, K. J. (1997). Distributed representations of structure: A theory of analogical access and mapping. Psychological Review, 104, 427-466.

Hummel, J. E., \& Holyoak, K. J. (2003). A symbolic-connectionist theory of relational inference and generalization. Psychological Review, 110, 220-264.

Krüger, F., Nuthmann, A., \& van der Meer, E. (2001). Pupillometric indices of temporal order representation in semantic memory. Zeitschrift für Psychologie, 209, 402-415.

LIEN, Y., \& CHENG, P. W. (2000). Distinguishing genuine from spurious causes: A coherence hypothesis. Cognitive Psychology, 40, 87-137.

Moss, H. E., Ostrin, R. K., Tyler, L. K., \& Marslen-Wilson, W. D. (1995). Accessing different types of lexical semantic information: Evidence from priming. Journal of Experimental Psychology: Learning, Memory, \& Cognition, 21, 863-883.

MurPHy, G. L. (2002). The big book of concepts. Cambridge, MA: MIT Press.

Murphy, G. L., \& Medin, D. L. (1985). The role of theories in conceptual coherence. Psychological Review, 92, 289-316.

Nelson, D. L., McEvoy, C. L., \& Schreiber, T. A. (1998). The University of South Florida word association, rhyme, and word fragment norms. Available at w3.usf.edu/FreeAssociation/.

Pearl, J. (2000). Causality: Models, reasoning, and inference. Cambridge: Cambridge University Press.

REHDER, B. (2003). A causal-model theory of conceptual representation and categorization. Journal of Experimental Psychology: Learning, Memory, \& Cognition, 29, 1141-1159.

Rinck, M., Hahnel, A., \& Becker, G. (2001). Using temporal information to construct, update, and retrieve situation models of narratives. Journal of Experimental Psychology: Learning, Memory, \& Cognition, 27, 67-80.

Shanks, D. R., \& Dickinson, A. (1987). Associative accounts of causality judgment. In G. H. Bower (Ed.), The psychology of learning and motivation (Vol. 21, pp. 229-261). New York: Academic Press.

Shanks, D. R., \& López, F. J. (1996). Causal order does not affect cue selection in human associative learning. Memory \& Cognition, 24, 511-522.

Spellman, B. A., Holyoak, K. J., \& Morrison, R. G. (2001). Analogical priming via semantic relations. Memory \& Cognition, 29, 383-393.

Tulving, E. (1972). Episodic and semantic memory. In E. Tulving \& W. Donaldson (Eds.), Organization and memory (pp. 381-403). New York: Academic Press.

Tyler, L. K., \& Moss, H. E. (1997). Functional properties of concepts: Studies of normal and brain-damaged patients. Cognitive Neuropsychology, 14, 511-545.

VAN DER MEer, E. (1999). Zeitkodierung in Wissenskörpern. Zeitschrift für Psychologie, 207, 363-381.

van Der Meer, E., Beyer, R., Heinze, B., \& Badel, I. (2002). Temporal order relations in language comprehension. Journal of Experimental Psychology: Learning, Memory, \& Cognition, 28, 770-779.

Waldmann, M. R. (1996). Knowledge-based causal induction. In D. R. Shanks, K. J. Holyoak, \& D. L. Medin (Eds.). The psychology of learning and motivation: Vol. 34. Causal learning (pp. 47-88). San Diego: Academic Press.

WALDMANN, M. R. (2000). Competition among causes but not effects in predictive and diagnostic learning. Journal of Experimental Psychology: Learning, Memory, \& Cognition, 26, 53-76.

Waldmann, M. R. (2001). Predictive versus diagnostic causal learning: Evidence from an overshadowing paradigm. Psychonomic Bulletin \& Review, 8, 600-608.

Waldmann, M. R., \& Holyoak, K. J. (1992). Predictive and diagnostic learning within causal models: Asymmetries in cue competition. Journal of Experimental Psychology: General, 121, 222-236.

Waldmann, M. R., Holyoak, K. J., \& Fratianne, A. (1995). Causal models and the acquisition of category structure. Journal of Experimental Psychology: General, 124, 181-206.

ZwaAn, R. A, \& YAXley, R. H. (2003). Spatial iconicity affects semantic relatedness judgments. Psychonomic Bulletin \& Review, 10, 954958 . 


\section{NOTE}

1. In an additional study, we tested the same causal stimuli in a within-subjects block design with 24 participants. Prior to each block, participants were prompted to perform either a causal or an associative evaluation of the word pairs. In different blocks we presented predictive, diagnostic, or noncausal associated item pairs along with a small number of filler items. The number of stimuli was the same for each block. The results yielded a significant difference in the causal block $[t(23)=2.53, p=.02]$, with a retrieval advantage for the predictive causal stimuli; no difference in the associative block $[t(23)=.5, p=$ $.62]$; and a marginally significant interaction $[t(23)=1.14, p=.13$, using a one-tailed criterion of 0.1$]$. The power of the causal items in the associative block was .85 .

\begin{abstract}
APPENDIX
Table A1

Normed Causal and Associative Filler Word Pairs Used in the Experiments

FSA and BSA indicate the forward and the backward strength of association in the USF data base (Nelson et al., 1998), respectively; nn refers to not normed. FD is the difference of the statistical frequency ratings in our norming study. Positive values indicate a higher estimated statistical frequency for the predictive direction, whereas negative values indicate a higher estimated frequency for the diagnostic direction. Word pairs marked with an asterisk were excluded in Experiments 2 and 3.
\end{abstract}

\begin{tabular}{|c|c|c|c|c|c|c|c|c|}
\hline \multicolumn{2}{|c|}{ Causal Word Pairs } & \multirow{2}{*}{$\frac{F D}{-12}$} & \multirow{2}{*}{$\begin{array}{c}\text { FSA } \\
.01\end{array}$} & \multirow{2}{*}{$\begin{array}{c}\text { BSA } \\
\mathrm{nn}\end{array}$} & \multicolumn{2}{|c|}{ Associative Word Pairs } & \multirow{2}{*}{$\begin{array}{c}\text { FSA } \\
.02\end{array}$} & \multirow{2}{*}{$\begin{array}{c}\text { BSA } \\
.00\end{array}$} \\
\hline absence & withdrawal & & & & acrobat & athletes & & \\
\hline acid & corrosion $^{*}$ & 5 & .00 & $\mathrm{nn}$ & agency & firm & .02 & .00 \\
\hline alcohol & accident & -4 & .00 & .00 & ambulance & rush & .01 & .00 \\
\hline attack & defense & 1 & .05 & .00 & antelope & gazelle & .03 & .03 \\
\hline bacteria & infection & 0 & .01 & .00 & atlas & dictionary & .01 & .00 \\
\hline bang & deafness & -11 & .00 & nn & basketball & teams & .00 & .02 \\
\hline beat & bruise & 8 & .00 & .05 & bedroom & furniture & .01 & .00 \\
\hline betrayal & distrust & 13 & .00 & .00 & caffeine & mountain & .01 & .00 \\
\hline birthrate & population & 1 & nn & .00 & car & plane & .01 & .03 \\
\hline carcinogen & tumor & 2 & $\mathrm{nn}$ & .00 & chipmunks & acorn & .00 & .01 \\
\hline chromosome & gender & 2 & .03 & .00 & claw & dogs & .01 & .00 \\
\hline compliment & blush & -4 & .00 & .00 & cocktails & fruits & .00 & .01 \\
\hline crime & arrest & -20 & .00 & .02 & computer & apple & .01 & .02 \\
\hline crush & damage & 3 & .00 & .01 & control & volume & .00 & .01 \\
\hline dairy & diarrhea & -7 & .00 & $\mathrm{nn}$ & dagger & fight & .01 & .00 \\
\hline diet & hunger & 16 & .01 & .00 & decency & respect & .03 & .00 \\
\hline disease & injection & 13 & .00 & .01 & elephant & zebra & .00 & .01 \\
\hline drought & famine & -2 & .00 & .02 & elevator & floor & .01 & .00 \\
\hline drug & relief & 23 & .00 & .00 & email & attachment & $\mathrm{nn}$ & .00 \\
\hline education & career & -7 & .00 & .03 & engine & roar & .02 & .00 \\
\hline espionage & treason* & -4 & .00 & .00 & envy & admire & .03 & .03 \\
\hline eyedrops & dilation & -20 & $\mathrm{nn}$ & .00 & family & sibling & .00 & .03 \\
\hline fertilizer & growth & 15 & $\mathrm{nn}$ & .00 & forecast & weather & $\mathrm{nn}$ & .00 \\
\hline fracture & cast & 12 & .00 & .00 & girl & maid & .00 & .01 \\
\hline frequency & pitch & -3 & .00 & .00 & glands & pituitary & .03 & nn \\
\hline frowning & wrinkles & 24 & .02 & .00 & glass & window & .01 & .02 \\
\hline gang & riot & -6 & .00 & .00 & grab & pull & .03 & .11 \\
\hline gases & explosion & -24 & .00 & .00 & graduation & gown & .02 & .00 \\
\hline genes & baldness* & -10 & .00 & .00 & graph & numbers & .02 & .00 \\
\hline gold & wealth & 7 & .02 & .00 & ground & potatoes & .00 & .02 \\
\hline hormones & mood & 4 & .00 & .00 & harbour & seaman & .00 & $\mathrm{nn}$ \\
\hline humidity & sweat & 27 & .02 & .00 & insurance & estimate & .00 & .01 \\
\hline illness & treatment & 12 & .00 & .04 & kill & theft & .00 & .01 \\
\hline invitation & visit & 10 & .00 & .00 & kindness & sympathy & .00 & .02 \\
\hline joke & amusement & -1 & .00 & $\mathrm{nn}$ & Latin & medicine & .02 & .00 \\
\hline lamp & heat & -3 & .00 & .00 & lettuce & vegetables & .08 & .00 \\
\hline lesion & scar & 7 & $\mathrm{nn}$ & .00 & lime & corona & .02 & $\mathrm{nn}$ \\
\hline lightning & fire & 4 & .00 & .00 & lover & girlfriend & .03 & .02 \\
\hline magnet & attraction & 6 & .10 & .04 & money & groceries & .00 & .02 \\
\hline moon & tide & -3 & .00 & .02 & mother & wife & .00 & .03 \\
\hline movie & nightmare & 0 & .00 & .02 & newspaper & gossip & .00 & .01 \\
\hline mutation & cancer & -19 & nn & .00 & office & employment & .00 & .02 \\
\hline nuts & allergy & -9 & .00 & .00 & ounce & gallon & .02 & .00 \\
\hline
\end{tabular}


APPENDIX (Continued)

\begin{tabular}{|c|c|c|c|c|c|c|c|c|}
\hline \multicolumn{9}{|c|}{ Table A1 (Continued) } \\
\hline \multicolumn{2}{|c|}{ Causal Word Pairs } & \multirow{2}{*}{$\frac{\text { FD }}{-9}$} & \multirow{2}{*}{$\begin{array}{c}\text { FSA } \\
.00\end{array}$} & \multirow{2}{*}{$\begin{array}{c}\text { BSA } \\
.00\end{array}$} & \multicolumn{2}{|c|}{ Associative Word Pairs } & \multirow{2}{*}{$\begin{array}{c}\text { FSA } \\
.05\end{array}$} & \multirow{2}{*}{$\begin{array}{r}\text { BSA } \\
.02\end{array}$} \\
\hline order & delivery & & & & painting & wall & & \\
\hline pain & aggression* & 10 & .00 & $\mathrm{nn}$ & paper & envelope & .00 & .03 \\
\hline panic & escape & -3 & .00 & .00 & patty & hamburger & $\mathrm{nn}$ & .02 \\
\hline period & cramps & 4 & .00 & .07 & planter & farmer & $\mathrm{nn}$ & .02 \\
\hline pollution & asthma & -7 & $\mathrm{nn}$ & .00 & power & voltage & .01 & .02 \\
\hline pressure & bursting & -22 & .00 & .00 & propeller & helicopter & $\mathrm{nn}$ & .03 \\
\hline sadness & crying & -20 & .05 & .13 & protestants & baptist & .00 & .01 \\
\hline salt & thirst & 21 & .00 & .00 & ring & emerald & .00 & .03 \\
\hline scratch & blood & -7 & .01 & .00 & round & screw & .00 & .01 \\
\hline shampoo & tears & -6 & .00 & .00 & sandwich & tomatoes & .00 & .00 \\
\hline shock & scream & 3 & .00 & .00 & security & force & .01 & .00 \\
\hline spice & flavor & -24 & .05 & .02 & session & course & .01 & .00 \\
\hline spill & stain & -7 & .00 & .00 & shape & curve & .01 & .02 \\
\hline sprain & swell & 18 & .00 & $\mathrm{nn}$ & shrimp & ocean & .03 & .00 \\
\hline stress & fatigue & 6 & .00 & .02 & soup & cracker & .03 & .04 \\
\hline study & pass & 6 & .00 & .00 & spray & roach & .03 & .03 \\
\hline sunlight & freckles & 5 & .00 & .02 & story & passage & .00 & .00 \\
\hline sweets & cavity & -27 & .00 & .00 & terms & meaning & .01 & .00 \\
\hline training & fitness & 12 & .00 & .00 & test & hypothesis & .00 & .03 \\
\hline trash & stink & 20 & .00 & .00 & towers & skyscraper & .02 & .05 \\
\hline trauma & coma & -9 & .01 & .00 & tuba & saxophone & .03 & .01 \\
\hline UV light & tanning & -9 & $\mathrm{nn}$ & nn & umbrella & tote & .00 & .01 \\
\hline vacuum & suction & 14 & .20 & $\mathrm{nn}$ & uniforms & officers & .00 & .01 \\
\hline virus & epidemic & -24 & .00 & $\mathrm{nn}$ & vehicle & bicycle & .02 & .01 \\
\hline wind & erosion & 10 & .00 & $\mathrm{nn}$ & vessel & vein & .01 & .02 \\
\hline \multicolumn{2}{|c|}{ Sum of rating differences } & 0 & & & & & & \\
\hline \multicolumn{2}{|c|}{ Mean strength of association } & & .01 & .01 & \multicolumn{2}{|c|}{ Mean strength of association } & .01 & .01 \\
\hline
\end{tabular}

Table A2

Unrelated Filler Word Pairs Used in Experiment 3

\begin{tabular}{|c|c|c|c|c|c|}
\hline \multicolumn{2}{|c|}{ Unrelated Word Pairs } & \multicolumn{2}{|c|}{ Unrelated Word Pairs } & \multicolumn{2}{|c|}{ Unrelated Word Pairs } \\
\hline ambulance & window & girl & agriculture & patty & kitchen \\
\hline ankle & farming & glands & sailor & phone & switch \\
\hline archer & phonebook & glass & rush & planter & power \\
\hline basin & academy & grab & screw & point & queen \\
\hline basket & kite & grass & fist & posters & hamburger \\
\hline beauty & compass & gymnastic & vegetables & potatoes & insult \\
\hline bedtime & tomatoes & harbor & garage & printer & angel \\
\hline brush & dices & insurance & ice cream & radiation & jockey \\
\hline bubble & velvet & investor & priming & report & bike \\
\hline caffeine & sky & kill & clock & revolting & roach \\
\hline chef & fear & king & ceiling & rise & bank \\
\hline clown & map & lamb & bearing & roles & cats \\
\hline conductor & groceries & landscape & maths & roof & seaman \\
\hline consulate & door & Latin & disgust & round & roar \\
\hline cookie & nose & lead & curve & salad & respect \\
\hline couch & dough & leather & pull & savage & airport \\
\hline dancer & liquid & lemon & soccer & security & floor \\
\hline deer & pencil & leopard & river & shape & aluminum \\
\hline diabetes & penny & lettuce & bars & ship & e-mail \\
\hline diamond & gear & medicine & passage & smock & plug \\
\hline disk & ground & mile & apron & spray & theft \\
\hline doorbell & architect & miracle & ginger & square & Indian \\
\hline drill & guest & money & piano & store & session \\
\hline eagle & child & mouse & light & story & dinner \\
\hline eggs & liar & mousepad & justice & survivor & cup \\
\hline elbow & pistol & mouth & actor & tea & graph \\
\hline elevator & force & needle & currency & therapy & barrel \\
\hline
\end{tabular}


APPENDIX (Continued)

\begin{tabular}{llllll}
\multicolumn{7}{c}{ APPENDIX (Continued) } \\
\hline \multicolumn{7}{c}{ Table A2 (Continued) } \\
\hline \multicolumn{7}{c}{ Unrelated } & Word Pairs & Unrelated & Word Pairs & Unrelated Word Pairs \\
\hline engine & glove & office & mirror & traffic & armrest \\
fabric & soup & onions & sphere & tree & maid \\
fairy & officers & page & tuxedo & truck & zebra \\
gate & cord & pants & bandage & voltage & mountain \\
gentlemen & chapter & parents & weather & water & boxer \\
\hline
\end{tabular}

(Manuscript received January 27, 2004;

revision accepted for publication October 4, 2004.) 